\title{
Incidental occult gunshot wound detected by postmortem computed tomography
}

\author{
Garyfalia Ampanozi • Nicole Schwendener • \\ Astrid Krauskopf • Michael J. Thali • \\ Christine Bartsch
}

Accepted: 18 July 2012/Published online: 6 September 2012

(C) Springer Science+Business Media, LLC 2012

\begin{abstract}
The body of a 59 year old woman underwent postmortem computed tomography (PMCT) examination prior to forensic autopsy, using a 256 slice multidetector row computed tomography scanner. A large left tension pneumothorax detected on the PMCT was considered to be a likely cause of death and this was confirmed at autopsy. In addition there was an unsuspected PMCT finding of a probable gunshot injury traversing the right orbit, facial bones and frontal sinus. The autopsy technique was adjusted accordingly and PMCT findings confirmed. PMCT in this case was not only diagnostic of cause of death, but also revealed retained projectile fragments of an old gunshot wound to the face. Without prior imaging such findings would have been undetected at autopsy. This case further underscores the contribution of routine PMCT examination to forensic autopsy practice.
\end{abstract}

Keywords Virtopsy · Postmortem

computed tomography $\cdot$ Gunshot $\cdot$ Pneumothorax

\section{Introduction}

Postmortem computed tomography (PMCT) is implemented in forensic examinations and is very helpful in detecting fluid, gas and metal objects [1,2]. The usefulness of PMCT in gunshot cases has been widely discussed

G. Ampanozi ( $\square)$ - N. Schwendener · A. Krauskopf .

M. J. Thali · C. Bartsch

Department of Forensic Medicine and Imaging, Institute of Forensic Medicine, University of Zurich, Winterthurerstrasse 190/52, 8057 Zurich, Switzerland

e-mail: garyfalia.ampanozi@irm.uzh.ch;

garyfalia.ampanozi@virtopsy.com
[3-9]. Compared to standard X-rays, it provides the significant advantage of determining the precise location of the projectile in three-dimensions [10], which is of great importance to the forensic pathologist.

Pneumothorax is defined as the presence of free air within the pleural space. It can be spontaneous (either with underlying lung disease or without), traumatic or iatrogenic [11, 12]. Spontaneous pneumothorax has been associated with emphysema-like changes and presence of bullae, more often located in the upper lungs [12]. There is evidence that spontaneous pneumothorax is associated with smoking and chronic obstructive pulmonary disease (COPD) [13, 14]. Mortality rates of pneumothorax are relatively low [15]. Detection of this pathologic entity is not easy at autopsy [16]. Moreover, proving the presence or absence of air in the pleura space during autopsies is not performed routinely in our institute, as a specific technique is required [17].

This case report presents two PMCT findings, one directly related to the cause of death (pneumothorax) and a second forensic relevant finding (gunshot wound), which underscore the potential contribution of PMCT in routine forensic autopsies.

\section{Case history}

A 59 year old woman was found dead by her husband in their residence, lying on a sofa. According to the husband, she was suffering from a chronic bronchitis and asthma; however, this information was never certified by a doctor or supported by her list of prescribed medication. The decedent's husband also reported, she was complaining of dyspnea for several days before she died. The decedent had a history of alcohol and nicotine abuse. No further information from her medical history was available. 


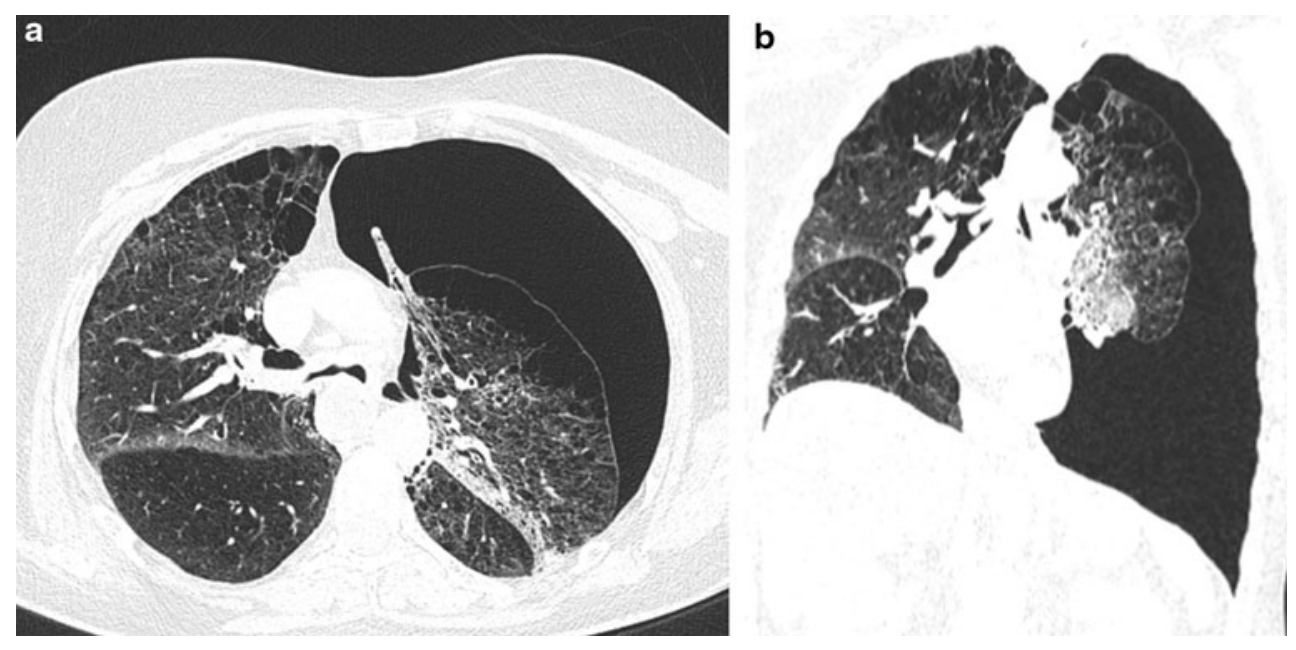

Fig. 1 a Axial and b coronal CT images through the thorax. Note the large left pneumothorax, mediastinal shift to the right, flattening of the left hemidiaphragm, emphysematous changes of the lung parenchyma, and the atelectatic area of the left upper lobe

\section{Scanning protocol}

The deceased was delivered to our Institute for further examination, as the cause and manner of death at external examination were undetermined. Prior to the autopsy, a whole body PMCT was performed using a 256 slice multidetector row computed tomography scanner (Somatom Definition Flash, Siemens Medical Solution, Forchheim, Germany). Whole body data acquisition was performed with the following technique: $120 \mathrm{kV}$; care dose mode; collimation $128 \times 0.6 \mathrm{~mm}$. Image reconstruction was carried out with a slice thickness of $1 \mathrm{~mm}$ at an increment of $0.6 \mathrm{~mm}$ (using both soft tissue and bone weighted reconstruction kernels). Axial, sagittal and coronal multiplanar reformations (MPR) as well as volume rendered (VR) images were created. Primary image review and 3D reconstructions were carried out on a CT workstation (Leonardo, Siemens medical solutions, Germany) and a picture archiving and communication system (IDS7, Sectra, Linköping, Sweden).

\section{Results}

Computed tomography findings

PMCT revealed a large left sided pneumothorax with mediastinal shift to the right and flattening of the left hemidiaphragm. Both upper lung lobes were emphysematous with multiple sizable bullae. Moreover, the left upper lobe showed atelectatic areas (Fig. 1).

No recent rib fractures were found. There was an old, healed fracture of the second left rib.

A second finding, unrelated to the cause of death, was that of numerous high density foreign bodies located at the medial and inferior wall of the right orbit, the frontal sinus, the maxilla and around the nasal bone (Fig. 2). The largest fragment had a maximum diameter of $10 \mathrm{~mm}$. Most of them were embedded in the osseous structures, but some were also found in the soft tissues. A mean Hounsfield units value of 3,070 suggested the fragments were metal. After careful examination, an old scar as well as a discontinuity of the platysma in the right submental area was detected. An osseous defect of the palate was also found, on the right side (Fig. 3).

\section{Autopsy findings}

The usual autopsy technique was adjusted based on the PMCT findings, as the following procedure is not performed routinely at our institute. After dissecting the thoracic soft tissues, the resulting tissue pockets were filled with water. Beneath the water surface an incision was made into the thoracic wall. On the left side, multiple air bubbles confirmed the presence of pneumothorax, as described in the literature [17]. Both lungs showed signs of COPD, such as chronic bronchitis and emphysema. The left side of the diaphragm was found to be considerably lowered, at the height of the seventh intercostal space. The old fracture of the second left rib was also confirmed.

In order to confirm the presence of the metal objects seen on PMCT, an extended dissection of the facial soft tissues was performed. After exposing the nasal bone and opening the frontal sinus, the foreign bodies were visualized (Fig. 4). The largest fragment was recovered and found to be compatible with a projectile fragment (Fig. 5). The scar in the right submental region could also be seen. It remains unclear whether this scar was also related to the old gunshot wound. 


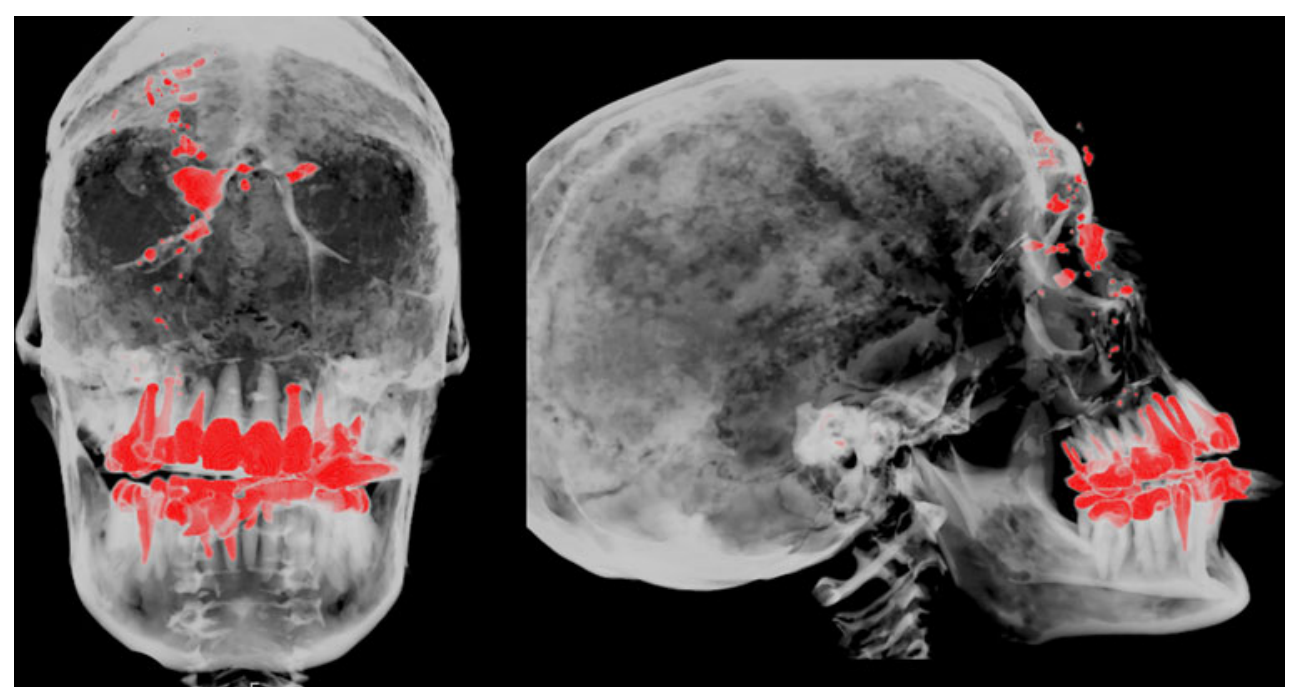

Fig. 2 3D maximum intensity projection of the skull. High density objects (dental work and foreign bodies) are colored red. Note the distribution of the foreign bodies around the bridge of the nose, the right medial orbital wall and the sinus frontalis

The cause of death was defined as right heart failure because of secondary spontaneous pneumothorax, following COPD-like changes of the lungs. The manner of death was natural. No further information regarding the old gunshot injury could be given by the husband or the police.

\section{Discussion}

Bolliger et al. [10] emphasize that PMCT is an invaluable tool for detecting foreign objects and gas, especially if the former are located in body parts that are not routinely dissected, such as the facial structures. This report is an excellent example of detecting both in the same case. We present this special case to show how PMCT can contribute significantly in recognizing pathological entities and direct the autopsy technique accordingly.

Although not forensically interesting, natural death cases are the largest proportion of the forensic pathologists workload [18]. Pneumothorax is securely documented only after performing a specific technique, which includes opening of the chest cavity under water [17]. This is probably the reason why cases of fatal pneumothorax described in the literature are accompanied by imaging examinations, such as X-rays $[19,20]$. In a study presented by Leth [21], out of 32 important PMCT findings missed at autopsy, 5 were pneumothoraxes and 2 projectiles.

In general, it is considered rare to incidentally find projectile fragments in living individuals [22]. Currently there is discussion in the literature as to the justification of removing them and what the complications for patients could be $[22,23]$. In the postmortem setting, such incidental findings are also seen. However, in the case reported

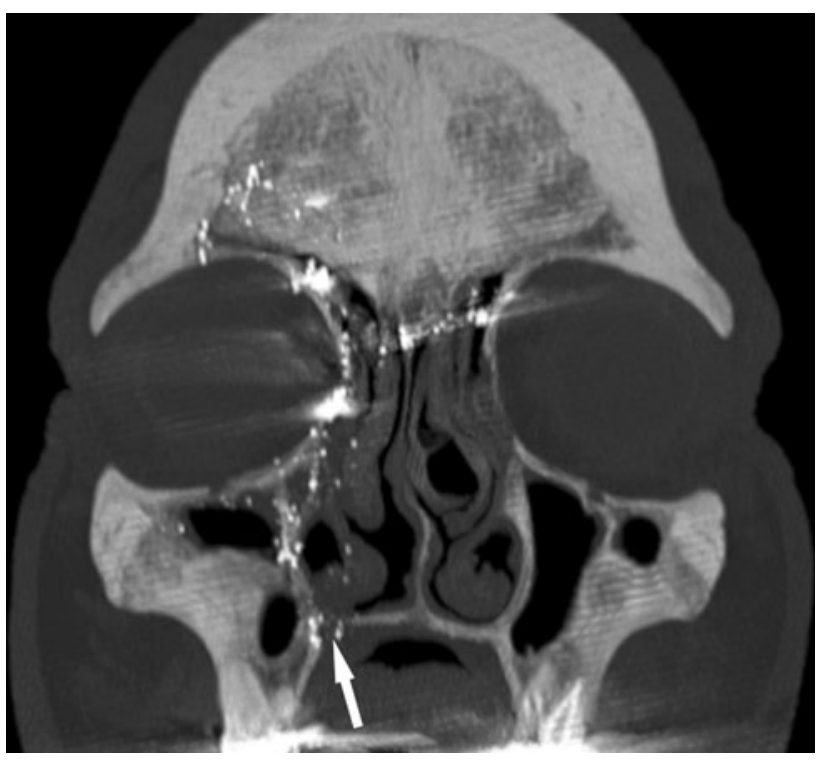

Fig. 3 Coronal maximum intensity projection. The metal fragments can be seen along the gunshot path. Note the osseous defect of the palate (arrow)

by Navarro et al. [24] an imaging modality (X-rays) was used as well.

The cause of death as well as the old gunshot injury to the head were both detected by PMCT and confirmed during autopsy. This case can be considered an example of how PMCT assists forensic investigations and the work of forensic pathologists, whose primary goal is to detect forensically relevant findings and to give clarity in each case. PMCT findings not only influenced the autopsy technique for precise confirmation and documentation of the cause of death, but also revealed a former gunshot 

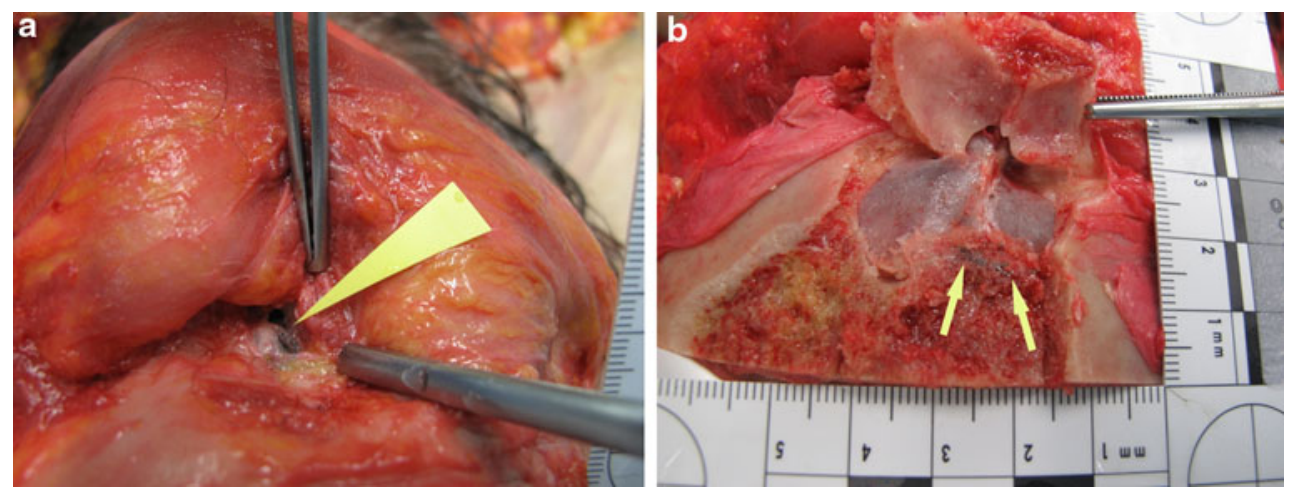

Fig. 4 Autopsy images confirming the presence of metallic foreign bodies. a The largest foreign body was found adjacent to the medial wall of the right orbit (arrowhead). b After opening the sinus frontalis, numerous small metal fragments were found (arrows)

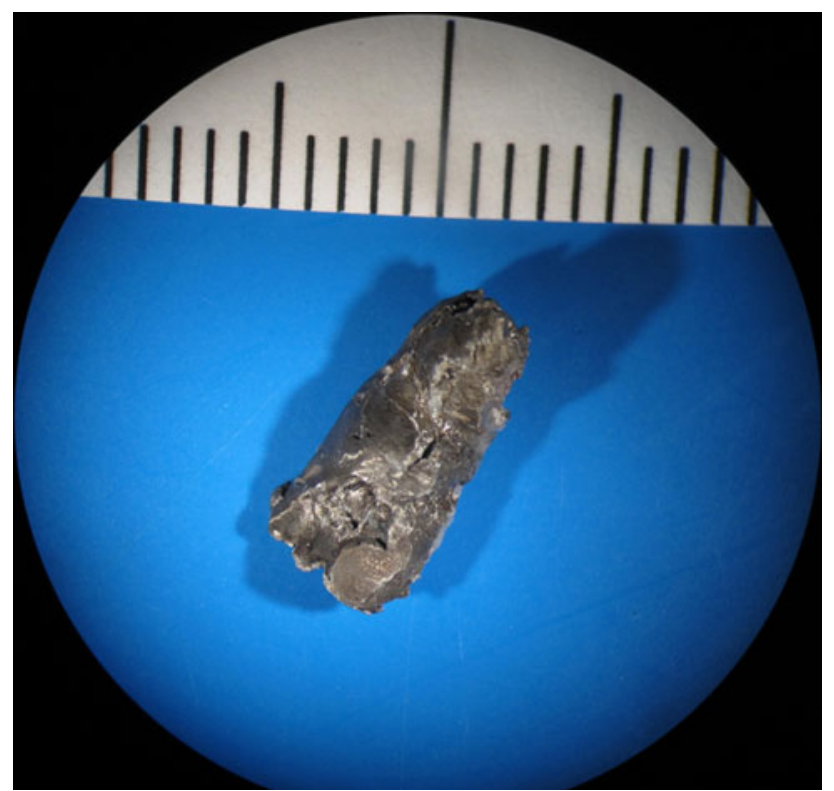

Fig. 5 The largest foreign body was examined and found to be compatible with a projectile fragment. Microscope photography $(\times 10)$

injury, which would have remained undetected if no radiological examination was performed.

\section{Key points}

1. Postmortem imaging determined the cause of death in this case as well as revealing an old gunshot injury.

2. Pneumothorax is an underreported cause of death if no radiological examination precedes autopsy.

3. Because of the imaging findings, the autopsy procedure could be adjusted to document the pneumothorax.

4. PMCT is an excellent tool for depicting foreign bodies and providing accurate localization of them.
Acknowledgments The authors would like to thank Gary Hatch for his significant contribution to the preparation of the manuscript.

\section{References}

1. Thali MJ, Dirnhofer R, Vock P. The virtopsy approach. 3D optical and radiological scanning and reconstruction in forensic medicine. Boca Raton: Taylor and Francis Group, LLC; 2009.

2. Thali MJ, Viner MD, Brogdon BG. Brogdon's forensic radiology. 2nd ed. Boca Raton: Taylor and Francis Group, LLC; 2011.

3. Levy AD, Abbott RM, Mallak CT, Getz JM, Harcke HT, Champion HR, Pearse LA. Virtual autopsy: preliminary experience in highvelocity gunshot wound victims. Radiology. 2006;240:522-8.

4. Thali MJ, Yen K, Vock P, Ozdoba C, Kneubuehl BP, Sonnenschein M, Dirnhofer R. Image-guided virtual autopsy findings of gunshot victims performed with multi-slice computed tomography (MSCT) and magnetic resonance imaging (MRI) and subsequent correlation between radiology and autopsy findings. Forensic Sci Int. 2003;138:8-16.

5. Berens S, Ketterer T, Kneubuehl BP, Thali MJ, Ross S, Bolliger SA. A case of homicidal intraoral gunshot and review of the literature. Forensic Sci Med Pathol. 2011;7:209-12.

6. Ruder TD, Ross S, Preiss U, Thali MJ. Minimally invasive post mortem CT-angiography in a case involving a gunshot wound. Leg Med (Tokyo). 2010;12:154-6.

7. Sano R, Hirawasa S, Kobayashi S, Shimada T, Awata S, Takei H, Otake H, Takahashi K, Takahashi Y, Kominato Y. Use of postmortem computed tomography to reveal an intraoral gunshot injuries in a charred body. Leg Med (Tokyo). 2011;13:286-8.

8. Stein KM, Bahner ML, Merkel J, Ain S, Mattern R. Detection of gunshot residues in routine CTs. Int J Legal Med. 2000;114:15-8.

9. Oehmichen M, Meissner C, König HG, Gehl HB. Gunshot injuries to the head and brain caused by low-velocity handguns and rifles. A review. Forensic Sci Int. 2004;146:111-20.

10. Bolliger SA, Thali MJ, Ross S, Buck U, Naether S, Vock P. Virtual autopsy using imaging: bridging radiologic and forensic sciences. A review of the virtopsy and similar projects. Eur Radiol. 2008;18:273-82.

11. Baumann MH. Pneumothorax. Semin Respir Crit Care Med. 2001;22:647-56.

12. Haynes D, Baumann MH. Pleural controversy: aetiology of pneumothorax. Respirology. 2011;16:604-10.

13. Cheng YL, Huang TW, Lin CK, Lee SC, Tzao C, Chen JC, Chang H. The impact of smoking in primary spontaneous pneumothorax. J Thorac Cardiovasc Surg. 2009;138:192-5. 
14. Bense L, Eklund G, Wiman LG. Smoking and the increased risk of contracting spontaneous pneumothorax. Chest. 1987;92:1009-12.

15. Gupta D, Hansell A, Nichols T, Duong T, Ayres JG, Strachan D. Epidemiology of pneumothorax in England. Thorax. 2000; 55:666-71.

16. Cha JG, Kim DH, Kim DH, Paik SH, Park JS, Park SJ, Lee HK, Hong HS, Choi DL, Yang KM, Chung NE, Lee BW, Seo JS. Utility of postmortem autopsy via whole-body imaging: initial observations comparing MDCT and 3.0 T MRI findings with autopsy findings. Korean J Radiol. 2010;11:395-406.

17. Saukko P, Knight B. Knight's forensic pathology. 3rd ed. London: Edward Arnold; 2004.

18. Christiansen LR, Collins KA. Natural death in the forensic setting. A study and approach to the autopsy. Am J Forensic Med Pathol. 2007;28:20-3.

19. Kurosaki K, Fushimi Y, Hara S, Kano S, Kuriiwa F, Nagai T, Endo T. Sudden death caused by tension pneumothorax after rupture of a thoracic aortic aneurysm case report. Am J Forensic Med Pathol. 2001;22:250-2.

20. Iwadate K, Ito H, Katsumura S, Matsuyama N, Sato K, Yonemura I, Ito Y. An autopsy case of bilateral tension pneumothorax after acupuncture. Leg Med (Tokyo). 2003;5:170-4.

21. Leth PM. Computerized tomography used as a routine procedure at postmortem investigations. Am J Forensic Med Pathol. 2009;30:219-22.

22. Kühnel TV, Tudor C, Neukam FW, Nkenke E, Stockmann P. Air gun pellet remaining in the maxillary sinus for 50 years: a relevant risk factor for the patient? Int J Oral Maxillofac Surg. 2010;39:407-11.

23. Wenham K, Craig S, Tsitrou E. The incidental finding of a retained gunshot pellet. Dent Update. 2009;36:28-30.

24. Navarro JA, Cortés E, Sanz CL, Pellicer MA. Finding of a bullet in the cervical column of a body hit by a train. J Forensic Sci. 1991;36:602-6. 